\title{
Surge Arrester Simulation in Fourier Transients Programs of Transmission Systems
}

\author{
A. U. M. AbDulazIZ and S. E. T. MOHAMED \\ Department of Electrical and Computer Engineering, Faculty of Engineering, \\ King Abdulaziz University, Jeddah, Saudi Arabia.
}

\begin{abstract}
The importance of simulating surge arresters in fast transients programs stems from their useful property as a means of controlling switching overvoltages in EHV and UHV systems. The paper proposes a surge arrester model to be used in conjunction with a transient program based on Fourier transform technique. The validity of the proposed model is demonstrated by computational results.
\end{abstract}

\section{Introduction}

The economics of bulk power transmission systems is largely influenced by the transmission system insulation cost. This tends to increase rapidly as operating voltages rise in the EHV and UHV ranges. Surge arresters have been introduced in transmission systems for purposes of limiting transient overvoltages and thereby reducing system insulation.

Surge arrester models in fast transients programs based on the Bewley lattice diagram method are well established. Recent developments have indicated the mertis of Fourier transform techniques in the analysis of such problems taking into consideration the frequency dependence of system parameters, such as circuit parameters of active and earth conductors and ground return path.

The present paper considers the simulation problem of surge arresters in a modified Fourier transients program. The arrester is simulated as a nonlinear element to comply with its volt-amp characteristic and time-varying nonlinearity. In the developed arrester model, the system boundary conditions at the arrester location are suitably modified to account for element flashover as well as arc extinction. Computational results are presented in the paper to demonstrate the validity of the developed simulation model. 


\section{Modified Fourier Transform for Fast Transients}

\subsection{General Form of the Transform}

A number of techniques have been proposed, based on the idea of travelling waves on transmission systems for the analysis of fast transients. Amongst these is the well established lattice diagram method put forward by Bewley ${ }^{[1]}$ and further developed by a number of investigators ${ }^{[2,3]}$. Other techniques have also been distinguished as being very useful in tackling the analysis of fast switching phenomena in transmission systems. Amongst these are Fourier transform and Laplace transform methods ${ }^{4]}$.

Fourier transform methods are particularly noted for their handling the frequency dependence of system parameters as some of these parameters encounter significant changes during the transient process. Fourier transform methods for transient phenomena are well established. The present paper aims at extending further the scope of these methods to analyze the problem of surge arresters in transmission systems.

The Fourier transform, $F(\omega)$, of a time varring function $f(t)$ is defined by the following integral

$$
F(\omega)=\int_{-\infty}^{+\infty} f(t) e^{-j \omega t} d t
$$

and the inverse transform $f(t)$ by the following integral

$$
f(t)=\frac{1}{\pi} \int_{-\infty}^{+\infty} F(\omega) e^{j \omega t} d \omega
$$

Any switching transient process may be analyzed by the Fourier transform technique over a certain period of time known as the observation time. At any instant in time the contribution of each frequency component in the Fourier expansion of the energizing voltage may be evaluated at any location on a transmission line. The time domain solution as shown by the inverse tramsform, equation (2), then represents the transient response to the energization process.

\subsection{The Modified Fourier Transform}

When considering energization of transmission systems, direct implementation of the Fourier transform pair is not possible. It is necessary to truncate both limits of integration of equation (1) for practical purposes, i.e., the integration should be conducted over a frequency range $(-\Omega, \Omega)$. Furthermore, since it is necessary to consider the system response over real time $(t \geq 0)$ and for a specified observation period $\left(T_{0}\right)$, equation (1) should be performed for $0 \leq t \leq T_{0}$.

It has been found that such practical limitations may be overcome in numerical evaluation of integrals (1) and (2) by making use of a modified version of the Fourier transform pair ${ }^{5,6]}$.

The modified transform avoids problems of poles on the integration path as it shifts this path in the complex $\omega$ plane by a suitable shift factor $a$, so that the path of integration is now $\operatorname{Real}(\omega)=a$ away from the ordinary path $\operatorname{Real}(\omega)=0$. Thus the 
modified Fourier transform becomes

$$
F(\omega-j a)=\int_{0}^{T_{0}} f(t) e^{-a t} e^{-j \omega t} d t
$$

and the inverse transform becomes

$$
f(t)=\frac{e^{a t}}{2 \pi} \int_{-\Omega}^{\Omega} F(\omega-j a) e^{j \omega t} d \omega
$$

When evaluating (3) and (4) numerically, it is necessary to make use of the fact that the Fourier transform $F(\omega)$ is complex for either a real or a complex time function $f(t)$. In order to effect economies on computer storage a number of half range expansions of the inverse integral may be utilized. A particular form has been found useful with a suitable choice of the shift factor $a$, based on trial and error. This form is

$$
f(t)=\operatorname{Real}\left[\frac{e^{a t}}{2 \pi} \cdot 2 \cdot \int_{-\Omega}^{\Omega} F(\omega-j a) e^{j \omega t} d \omega\right]
$$

The analysis proceeds by evaluating $F(\omega-j a)$ with a frequency step equal to twice the fundamental frequency $\omega_{0}$. Then either even or odd harmonics may be taken.

Considering odd harmonics only, equation (5) may be numerically evaluated for physical systems as follows

$$
f(t)=\frac{e^{a t}}{2 \pi} \cdot 2 \omega_{0} \cdot \text { Real }\left[\sum_{n=1}^{2 N-1} F\left(\omega_{n}-j a\right) e^{j \omega_{n} t}\right]
$$

where

$$
\begin{aligned}
& 2 N=\text { total number of harmonics selected for evaluating frequency response } \\
& \omega_{n}=(2 n-1) \omega_{0} \\
& \omega_{0}=\text { fundamental frequency } \\
& n=\text { order of harmonic selected. }
\end{aligned}
$$

It is worth noting that the choice of the step length $2 \omega_{0}$ increases the truncation frequency. However, this choice also helps reduce the number of summations for each time step. The fundamental frequency, $\omega_{0}$, is thus related to the observation time $T_{0}$, by

$$
\omega_{0}=\frac{\pi}{T_{0}}
$$

It is necessary to carry out a further modification of equation (5) by a suitable factor known as the sigma factor $(\sigma)$. The idea is that when the infinite time range is truncated, this influences adversely the rise time of $f(t)$ and also causes unstable oscillations, known as Gibbs oscillations. Mullinux ${ }^{[6]}$ introduced the idea of sigma factor in order to improve the situation. The sigma factor is defined as

$$
\sigma=\frac{\sin (2 n-1) \pi /(2 N-1)}{(2 n-1) \pi /(2 N-1)}
$$

Thus equation (6) now becomes after modification 


$$
f(t)=\frac{e^{a t}}{\pi} \cdot \omega_{0} \operatorname{Real}\left[\sum_{n=1}^{2 N-1} F\left(\omega_{n}-j a\right) e^{j \omega_{n} t} \cdot \sigma\right]
$$

\subsection{Fourier Transform of Time-Varying Nonlinearity}

Simulation of surge arresters in fast transients programs requires dealing with time varying nonlinearities. In order to handle such nonlinearities, Wedepohl and Mohamed ${ }^{[7]}$ proposed a useful algorithm based on piecewise synthesis. In their algorithm the time-varying function is linearized over a time step and the Fourier transform of the resultant decomposed strips of the original function are obtained. Thereafter the principle of superposition is applied and the entire transform is formulated.

In this paper use is made of the piecewise synthesis approach in order to simulate the response of the arrester in the frequency domain. This response provides for a new stimulus fed to the system at the arrester location to account for its history following the system disturbance.

\section{Surge Arrester Simulation}

\subsection{Basic Concepts}

A surge arrester model suited for inclusion in a fast transients program should be capable of reproducing faithfully the true characteristics of a surge arrester. In real time, a surge arrester should not pass any current at normal operating conditions. It should breakdown at specified levels of transient overvoltages, discharging the transient surge to ground. Furthermore the arrester should interrupt power-follow currents that may cause permanent faults in the system ${ }^{[8.9]}$.

In order to achieve this characteristic a surge arrester has a nonlinear form for voltage dependent nonlinearity. This is defined as

where

$$
I_{a}=K V_{a}^{\alpha}
$$

$I_{a}=$ discharge arrester current

$V_{a}=$ applied voltage across arrester terminals

$K$ and $\alpha$ are constants depending on material and dimension of element. ( $\alpha$ varies between 2 and 6 ).

\subsection{Arrester Model Algorithm}

Prior to arrester sparkover, the system transient overvoltage is computed at the arrester location following line energization. The information is stored in the computer memory and then processed using piecewise synthesis method in order to obtain its modified Fourier transform components. In addition the approximate line surge impedance $\left(Z_{0}\right)$ looking from the direction of the arrester location is also obtained. This represents the line input impedance at the surge arrester terminals. Thus at the arrester location a Thevenin source is obtained with a time varying voltage $\left(V_{0}\right)$. Figures 1 and 2 show a typical transmission network suited for fast transient studies and also its equivalent circuit from which the actual transient overvoltage at the arrester termi- 
nals may be determined. Thus

$$
V_{a}=V_{0}-Z_{0} I_{a}
$$

From (8) and (9) both $V_{a}$ and $I_{a}$ may be determined. Therefore

$$
K^{\prime} I_{a}^{\beta}+Z_{0} I_{a}-V_{0}=0
$$

where

$$
\begin{aligned}
& \beta=1 / \alpha \\
& K^{\prime}=K^{-\beta}
\end{aligned}
$$

When the arrester voltage and current are determined from (10), suitable injection of these quantities will then account for the existence of the arrester and its role as a surge limiting device. Later, when the arrester current passes through zero the arrester model will be removed from the circuit. If the overvoltage at the surge arrester terminal exceeds the limiting condition once more, another flashover will take place in order to reduce the newly produced overvoltage. Theoretically the process will continue repeating itself, but practically the circuit damping and losses will bring it to an early end.

It is worthy of note that the solution of equation (10) may effected numerically using standard numerical techniques.

\subsection{Mathematical Model for Arrester Simulation}

Figure 1 shows a single line diagram for a transmission system energized from a switching station. A surge arrester is located at the end of the line. Under steady state conditions and before flashover the line matrix nodal equation may be written as

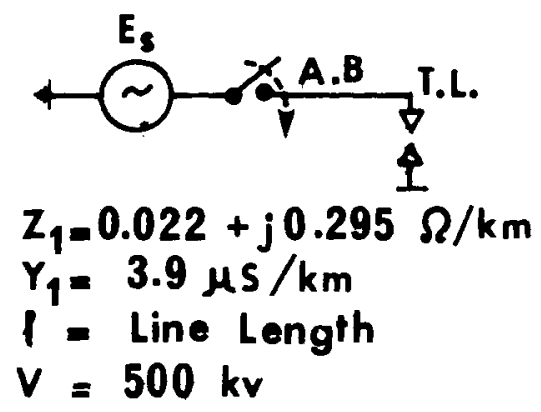

Fig. la. System diagram.

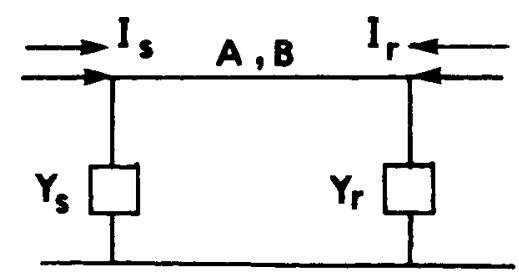

FIG. Ib. System equivalent circuit.

$$
\left[\begin{array}{c}
I_{s} \\
I_{r}
\end{array}\right]=\left[\begin{array}{cc}
A+Y_{s} & -B \\
-B & A+Y_{r}
\end{array}\right]\left[\begin{array}{c}
V_{s} \\
V_{r}
\end{array}\right]
$$


where

$V_{s}, V_{r}$ are sending and receiving end voltages,

$I_{s}, I_{r}$ are sending and receiving end currents,

$A=Y_{0}$ coth $\gamma \ell, \quad B=Y_{0} \operatorname{cosech} \gamma \ell$,

$Y_{s}$ and $Y_{r}$ are system terminal admittances,

$Y_{0}$ is line surge admittance,

$\gamma$ is line propagation constant,

$\ell$ is line length.

As arrester has not flashed over $I_{r}=0$, upon solution $V_{r}$ and $V_{s}$ may be determined Thus

$$
\begin{aligned}
V_{r} & =\frac{B}{\left(A+Y_{r}\right)} V_{s} \\
I_{s} & =Y_{s} E_{s} \\
V_{s} & =\frac{Y_{s} E_{s}}{\left[\left(A+Y_{s}\right)-B^{2} /\left(A+Y_{r}\right)\right]}
\end{aligned}
$$

When the arrester flashes over, the input impedance at the arrester location is equivalent to the line surge impedance. The nodal matrix equation representing this condition is

$$
\left[\begin{array}{cc}
Y_{s} & E_{s} \\
Y_{0} & V_{0}
\end{array}\right]=\left[\begin{array}{cc}
A+Y_{s} & -B \\
-B & A+Y_{0}
\end{array}\right]\left[\begin{array}{c}
V_{s} \\
Y_{r}
\end{array}\right]
$$

upon solution

$$
\begin{aligned}
& V_{r}=\frac{Y_{0} V_{0}+B V_{s}}{A+Y_{0}} \\
& V_{s}=\frac{Y_{s} E_{s}\left(A+Y_{0}\right)+B Y_{0} V_{0}}{\left(A+Y_{s}\right)\left(A+Y_{0}\right)-B^{2}}
\end{aligned}
$$

It is worth noting that equations (13), (15) and (16) are equations in the frequency domain and that in order to obtain their time-domain solution, recourse is made to numerical solution of the inverse modified Fourier transform described by equation (7). The computational procedure to simulate the surge diverter is as follows 


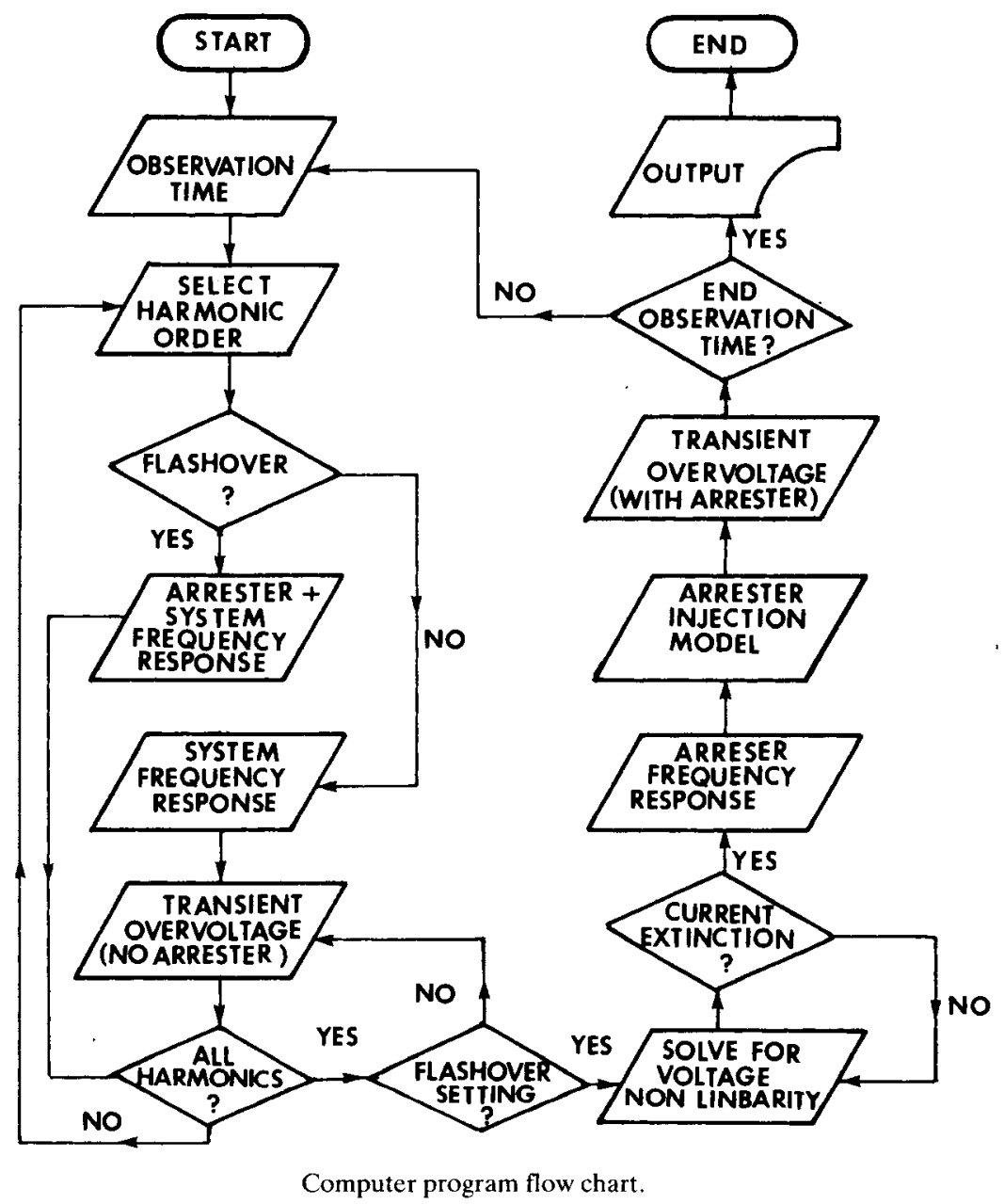

4. Computational Results and Discussion

A surge arrester model is chosen with a voltage dependent nonlinearity. The arrester is installed at the received end of a line energized at peak value of a sinusoid from a finite source. Both sending and receiving transient voltages are computed, and the results displayed in the figures shown. A single phase system is assumed for demonstration purposes (Fig. 2).

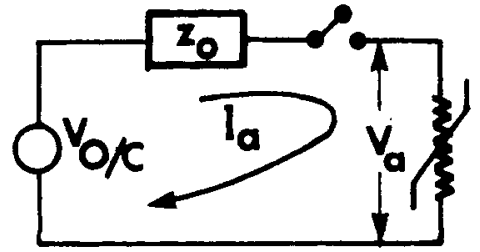

FIG. 2. Arrester equivalent circuit. 
Figure 3 shows sending end and receiving end transient voltages when the line is energized from a source of infinite capacity. The surge arrester in this case is assumed to flash over at voltages in excess of 2.0 p.u. The transient voltage at the arrester location is then computed at each time interval over the whole observation period of 10 $\mathrm{ms}$. This quantity represents the time dependent nonlinearity of the surge arrester assuming the flashover level stipulated.

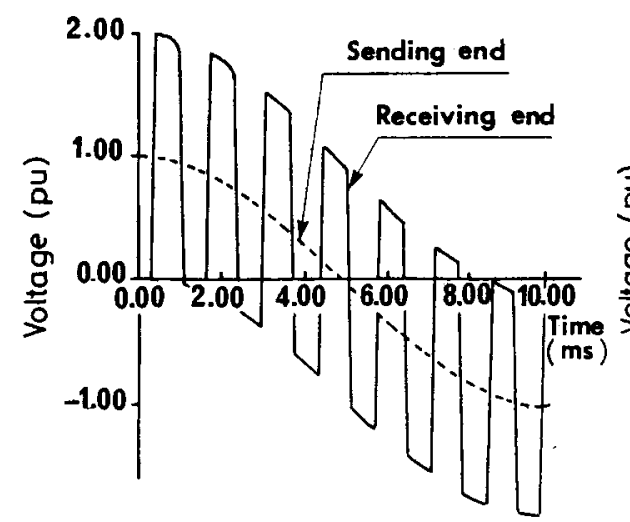

(a) Fourier

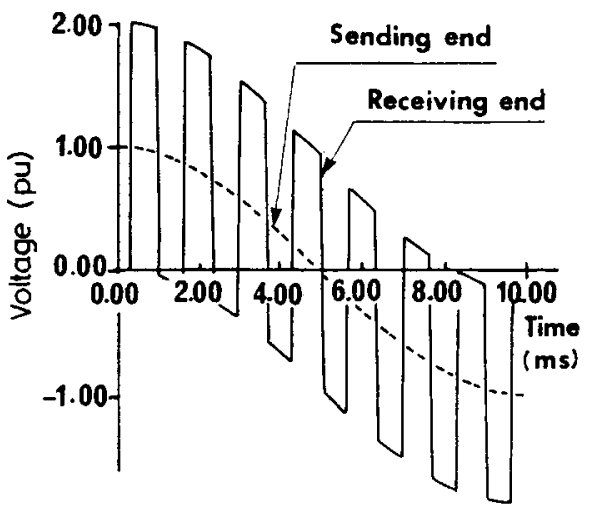

(b) Lattice

FIG. 3. Arrester inoperative (infinite bus, $\ell=100 \mathrm{~km}$ ).

As expected, Fig. 3a shows the results for this condition. The sending end voltage resembles that of the energizing voltage (except for a short rise time ${ }^{[5]}$ ). The received end voltage is characterized by the line natural oscillation. Clearly, the surge arrester remains inoperative as its setting is in excess of the maximum received end overvoltage. The result has been compared with a Bewley lattice diagram solution, Fig. 3b, and is found to be closely identical with it. This demonstrates the validity of the simulation method proposed for this special case.

Figure 4a shows receiving end overvoltages without surge arrester and when the surge arrester flashover level is set at $1.5 \mathrm{p}$.u. In latter case the line is energized from a finite source of 20000 MVA capacity and the transient response is observed for an approximate period of one power frequency cycle. At the received end it is seen that the system overvoltage has built up to $1.5 \mathrm{p.u}$., when the arrester current starts to flow, thereby causing the terminal overvoltage not to exceed the preset limit. The system overvoltage is seen to stay near the limit value for about $1.2 \mathrm{~ms}$, thereafter it starts to decrease gradually and is ultimately damped to its power frequency value. Figure $4 \mathrm{~b}$ shows a comparative result based on Bewley lattice solution. At the instant of flashover the arrester current jumps to about $1.0 \mathrm{kA}$. This current is further characterized by a slight jump due to the arrival of a reflected wave from the inductive source. The surge arrester remains engaged in the circuit until its current passes through zero as shown in Fig. 5a. The arrester current is interrupted at its first zero. Consequently it is switched off and kept inoperative, until the overvoltage at the arrester terminal exceeds the preset value again. However, the transient overvoltage is 
controlled by the arrester characteristic and it is seen that the terminal voltage has not shown any newly produced overvoltages.
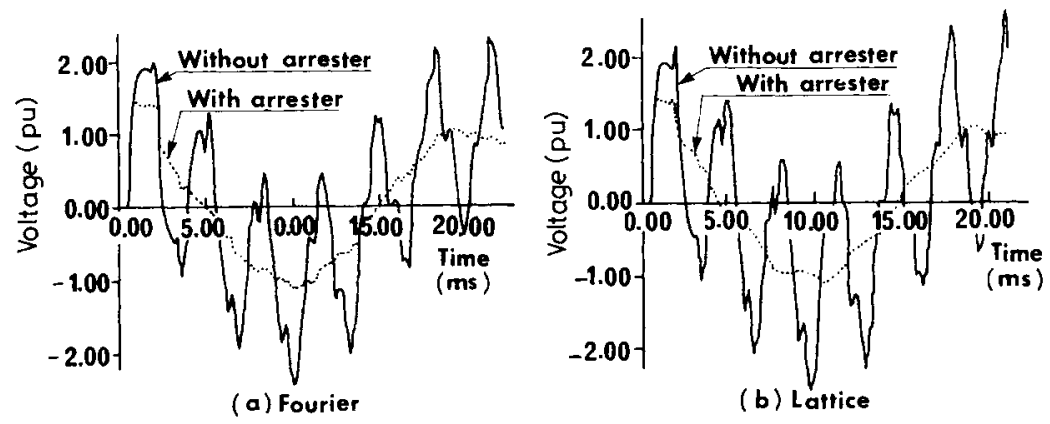

FIG. 4. Receiving end voltage with and without arrester (20000 MVA source, $\ell=200 \mathrm{~km})$.

Figure $5 \mathrm{~b}$ is a comparative result based on Bewley lattice solution.
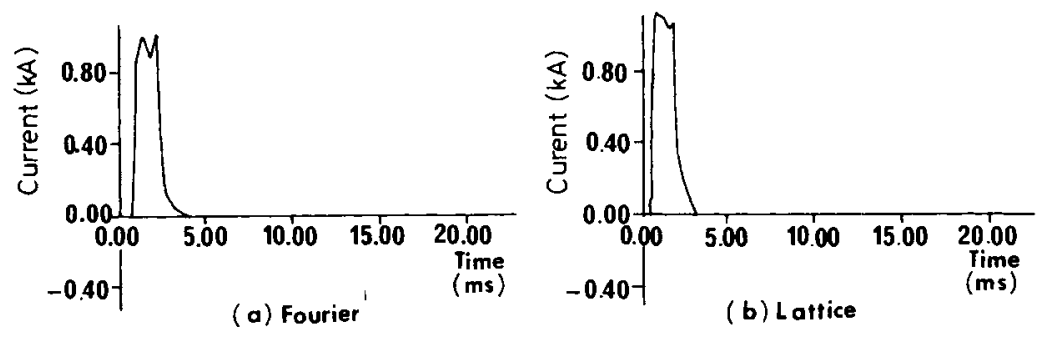

FIG. 5. Arrester flashover and extinction current.

As seen from the comparative results presented the surge arrester simulation based on Fourier technique compares favorably well with standard simulation based on Bewley lattice method. This is particularly noticeable in the terminal system voltage as the arrester engages in the circuit and controls the level of switching overvoltage at the termination. The marginal variation in the shape and magnitude of the voltage and current waveforms, however, is due to inaccuracies resulting from ignoring frequency dependance and distortion effects in the lattice technique.

\section{Conclusion}

The paper has highlighted the importance of surge arresters in limiting transient overvoltages in transmission systems. It has presented a proposed model for simulating these devices in fast transient programs that are based on Fourier transform techniques. These techniques are known to have distinct merits and thus the surge arrester model which takes into consideration both voltage-dependent and time-dependent nonlinearities, has helped extend their scope in handling such nonlinearities in transmission systems. The arrester model proposed considers both flashover and arc extinction characteristic of the surge arrester. Computational results presented in 
the paper have verified the model proposed as these results compare favorably well with those of typically standard techniques based on Bewley lattice methods.

\section{References}

[1] Bewley, L.V., Travelling Waves on Transmission Systems, Dover Publications, New York, U.S.A. (1963).

[2] Barthold, L.O. and Carter, J.K., Digital travelling-wave solutions (I - Single-phase equivalents), AIEE Trans., PAS-80: 812 (1961).

[3] Bickford, J.P. and Doepal, P.S., Calculation of switching transients with particular reference to line energization, IEE Proc. 114 (4): 465 (1967).

[4] Dommel, H.W., Digital computers solution of electromagnetic transients in single- and multi-phase networks, IEEE Trans., PAS-88: 388 (1969).

[5] Mullinux, Day, Sylvia, Reed and John, Developments in obtaining transient response using Fourier transforms: Gibbs phenomena and Fourier integrals, Internat. J. Elect. Eng. Educ. 3: 501-506(1965).

[6] Mullinux, Day, Sylvia, Reed and John, Development in obtaining transient response using Fourier transforms: Use of the modified Fourier transforms, ibid., 4: 31-40 (1966).

[7] Wedepohl, L.M. and Mohamed, S.E.T., Transient analysis of multiconductor transmission lines with special reference to non-linear problems, Proc. IEE, 117 (5): (1970).

[8] Bickford, J.P., et al., Dynamic model of a surge arrester for computer calculation of lightning performance of substations, Proc. IEE, 131 (7) Pt.C: $357-362$ (1984).

[9] Torres, C.R., Prediction of lightning performance of zinc oxide arresters on distribution lines, IEE, Lightning and Power Systems Conference Publication, No. 236, pp. 81-85 (1984). 


$$
\text { محاكاة كامـح الأمـواج العارمـة في بوامـج الفوريسير لتحليـل الظـواهر العـابرة }
$$

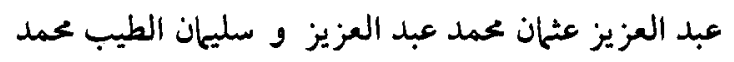

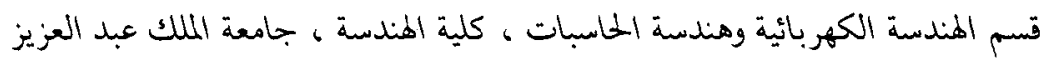

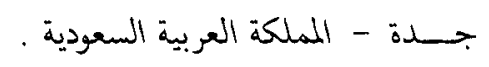

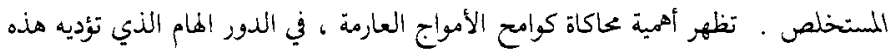

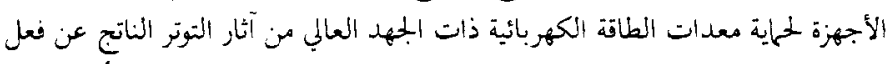

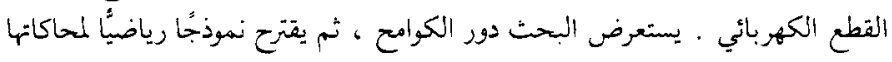

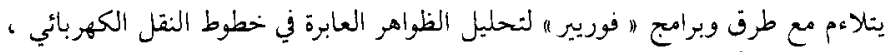

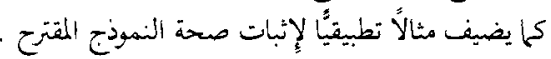

